\title{
Mutations of filament-aggregating protein gene in Romanian children diagnosed with atopic dermatitis
}

\author{
ANCA E. CHIRIAC ${ }^{1,2^{*}}$, ROXANA POPESCU ${ }^{3}$, LĂCRĂMIOARA BUTNARIU ${ }^{3}$, \\ ALINA MURGU $^{4}$, LILIANA FOIA ${ }^{5^{*}}$ and DOINA AZOICAI ${ }^{2 *}$ \\ ${ }^{1}$ Department of Dermatology, Nicolina Medical Center, 700613 Iasi; ${ }^{2}$ Department of Epidemiology, \\ 'Grigore T. Popa' University of Medicine and Pharmacy; ${ }^{3}$ Department of Genetics, 'Sfânta Maria' Emergency \\ Clinical Hospital for Children, Regional Center for Medical Genetics, 'Grigore T. Popa' University of Medicine \\ and Pharmacy; ${ }^{4}$ Department of Pediatrics, 'Sfânta Maria' Emergency Clinical Hospital for Children, 'Grigore T. Popa' \\ University of Medicine and Pharmacy; ${ }^{5}$ Department of Biochemistry, Faculty of Dental Medicine, \\ ‘Grigore T. Popa' University of Medicine and Pharmacy, 700115 Iasi, Romania
}

Received July 10, 2020; Accepted August 11, 2020

DOI: $10.3892 /$ etm.2020.9343

\begin{abstract}
Association of atopic dermatitis (AD) and several mutations of various genes of the immune system, in particular filament-aggregating protein gene (FLG) has been investigated in many studies. The association between defective FLG and $\mathrm{AD}$ in the Romanian population has not been assessed or published. The present study focused on the genetic background of $\mathrm{AD}$, aiming to assess the prevalence of FLG mutations in Romanian patients with AD. Genetic background of AD was tested for common FLG-mutations: R501X, 2282del4, S3247X and R2447X. The study involved 48 Romanian Caucasian children aged between two months and six years diagnosed with AD, and 48 healthy volunteers; DNA extraction involved $50 \%$ of the patients to give samples by using buccal swabs and $50 \%$ by collection of whole blood samples. Genetic predisposition was evaluated based on family history, atopy history and profilaggrin genotyping. DNA extracted from blood samples was adequate to study FLG mutations, although no mutation was identified. Genetic factors do not have a unique critical role in AD; therefore, environmental factors unquestionably play an important role in this disease, but the clear-cut part that these factors trigger toward increasing the risk of $\mathrm{AD}$ in childhood is still obscure.
\end{abstract}

Correspondence to: Professor Liliana Foia, Department of Biochemistry, Faculty of Dental Medicine, 'Grigore T. Popa' University of Medicine and Pharmacy, 16 Universității Street, 700115 Iasi, Romania

E-mail: lilifoia@yahoo.co.uk

${ }^{*}$ Contributed equally

Abbreviations: FLG, filament-aggregating protein gene; AD, atopic dermatitis

Key words: FLG mutations, atopic dermatitis, Romanian patients, filaggrin genotyping, DNA sampling, DNA extraction

\section{Introduction}

Atopic dermatitis (AD, atopic eczema) is a chronic inflammatory skin disease, intensely pruritic, with an atopic background, that has a great impact on the quality of life of the patients and members of their family. Epidemiologic studies have reported a marked variation (1-25\%) with a high prevalence for industrialized countries and a considerably lower one in Eastern Europe (1-3).

Stratum corneum, the outermost epidermal region, consists of cornified cells. This layer protects against dehydration, injury, pathogens and allergens. Stratum corneum is controlled by different genes, including the filament-aggregating protein gene (FLG) which encodes profilaggrin (the precursor of filaggrin). FLG is located in the epidermal differentiation complex, on chromosome 1 at locus 1q21 (4). Filaggrin is a key structural protein required for fully competent epidermal barrier function. In stratum corneum, filaggrin is degraded to amino acids; these contribute to the natural moisturizing factor (NMF) formation, which is essential for the hydration of stratum corneum (5) and protection of skin against the UVB radiation (6).

It has been proven that mutations of the FLG are associated with AD (7-9). FLG is highly polymorphic with 20 mutations reported in the European Caucasian population. FLG loss of-function (null) mutations were indicated to be associated with ichthyosis vulgaris and AD (10-14). The most frequent null mutations reported for European populations, R501X, 2282del4 and R2447X $(10,15)$, were associated with either reduced levels or absence of filaggrin and thus involving an affected skin barrier and decreased skin hydration. Mutation carriers had reduced levels of NMF compared with non-carriers, which explains the dry skin, the main feature of $\mathrm{AD}(16-18)$.

A few studies have reported a low prevalence of AD in Romania (19), but no data on the prevalence of FLG mutations in AD patients compared with non-AD patients are available. 


\section{Subjects and methods}

The study was conducted on 48 Romanian Caucasian children aged between two months and six years, who were diagnosed with AD accordingly to the criteria of Hannifin and Rajka (20). Gender and age-matched ( \pm 3 years) 48 healthy volunteers without history of ongoing or previous skin diseases were included as controls.

The study received ethics approval and consent by the institutional/national Ethics Committee of 'Grigore T. Popa' University of Medicine and Pharmacy, Iasi, Romania (Committee Reference no. 22168 of 10.10.2019). In accordance with the Declaration of Helsinki for all research involving human subjects, written informed consent for participation in the study and use of their tissue/blood/saliva sample (DNA sample) was obtained from all participants (or their parent or legal guardian in the case of children under 18 years of age).

A medical interview including questions about personal and familial antecedents of $\mathrm{AD}$ and an examination was performed by a dermatologist and a pediatrician. All other atopic diseases (asthma, allergic rhinitis and allergic conjunctivitis) were recorded, as well as lab investigations. Medical history and previous medical data were carefully examined and registered separately. The severity of AD was determined by the objective score of AD (OSCORAD) Index (21) and dichotomised according to OSCORAD values: mild (OSCORAD $<15$ ), moderate (OSCORAD 15-40) and severe (OSCORAD >40).

First part of the study was based on non-invasive methods of collecting DNA samples (buccal swabs), applied in order to identify the carriers of an FLG mutation in half of the patients enrolled in the study (24 AD patients and 24 normal controls). This procedure was carried out upon request from the parents to avoid the fear of needles of their children.

DNA sampling and extraction. Samples were obtained by using buccal swabs and sterile tubes for transportation. The samples were collected by specially trained physicians in the field (dermatologist or pediatrician). Care was taken that the samples collected from patients and volunteers were not contaminated with foreign DNA. For collecting the samples, it was ensured that the patient/control avoided eating, drinking or chewing gum for at least $30 \mathrm{~min}$ before; their mouth was rinsed with water. The sterile swab was extracted from the transport tube and rotated for $1 \mathrm{~min}$ on the inner side of the cheek mucosal, pushing slightly to the outside.

The method was gentle and caused no harm to any child and members of their respected family were present during sample collection. The swab was left to dry for $30 \mathrm{sec}$ up to $1 \mathrm{~min}$ and introduced directly to the original container/tube transport. The tubes were tightly closed. For each person enrolled in the study two samples were achieved, one for the inner side of each cheek. The tubes were labelled with personal data, stored at $2-8^{\circ} \mathrm{C}$ and sent immediately for DNA extraction.

Genomic DNA was extracted using a commercial DNA isolation kit based on a standard proteinase $\mathrm{K}$ digestion method, according to the manufacturer's protocol. The swabs were treated with a buffered Proteinase K solution, incubated at $55^{\circ} \mathrm{C}$ and then mixed with 96-100\% ethanol. The genomic DNA was purified using a spin column-based centrifugation procedure at $9,000 \mathrm{x}$ for $1 \mathrm{~min}$, after removal of the supernatant. The DNA was quantified by absorbance at $260 \mathrm{~nm}$, with a compact full spectrum UV-Vis instrument for rapid nucleic acid quantification in small volumes, and stored at $-20^{\circ} \mathrm{C}$.

Second part of the study was carried out using DNA extracted from whole blood sample (full vacutainer with EDTA) obtained from the other lot of patients enrolled in the study (24 patients diagnosed with AD and 24 volunteers). Similar analysis was performed and results were compared.

Filaggrin genotyping. The detection of FLG mutations relies on hybridization (annealing) of a short synthetic oligonucleotide (10-40 bases) to one or the other genomic DNA strand by means of restriction fragment length polymorphism (RFLP) or $5^{\prime}$ Nuclease (TaqMan) allele-specific RT-PCR. The RFLP analysis was performed following a PCR-based amplification (polymerase chain reaction) of a specific region of the FLG. RFLP makes use of different restriction endonucleases and their high affinity to cut unique and specific DNA sequences. By performing a restriction digest on a double-stranded DNA sample and assessing the resulting fragment lengths, it was determined whether a specific restriction site was present.

The DNA fragments were electrophoretically sized. The advantage of the method was that mutation detection not only relies on the hybridization specificity of the PCR primer but also on the restriction enzyme as well as the correct size. The 5'Nuclease (TaqMan) allele-specific RT-PCR method exploits the 5'nuclease activity of the Taq polymerase. The TaqMan assay was performed concurrently with a PCR reaction, and the results were read in real time. The assay used specific forward and reverse PCR primers that amplify a region that includes the mutation site.

Allele recognition was achieved using allele specific probes that hybridize specifically to the polymorphic site. The probes had a fluorescence resonance energy transfer (FRET) fluorophore at the 5 'end and a quencher molecule linked to their 3'end. When the probe was intact, the quencher was in close proximity to the fluorophore, preventing the fluorophore signal. During the PCR annealing/amplification step, the allele-specific probe bind to the target DNA strand and then is degraded by 5 'nuclease activity of the Taq polymerase as it extends the DNA from the PCR primers resulting in the separation of the fluorophore from the quencher molecule, thereby generating a detectable signal.

\section{Results}

The first method used to collect DNA samples using buccal swabs was a non-invasive, easy, relatively fast technique. Although very laborious for DNA extraction, the first method was not able to extract sufficient DNA in order to allow the study of FLG mutations. Therefore, out of a total of $48 \mathrm{AD}$ patients and 48 healthy volunteers included at the beginning of the study, genotyping was carried out in $50 \%$ of the children included: 24 volunteers and 24 diagnosed with AD.

DNA extracted from blood samples was adequate for study of FLG mutations, but no profilaggrin mutations R501X, 2282del4, S3247X and R2447X were identified in our batch. Samples were screened for population-specific FLG mutations by Sanger sequencing and then, the entire FLG was analyzed again with the smMIP-NGS strategy. 
In both studies, a similar number of healthy control individuals were tested and the results were also negative.

\section{Discussion}

The study focused on the genetic background of $\mathrm{AD}$, aiming to assess the FLG mutations R501X, 2282de14, S3247X and R2447X in Romanian patients.

The challenging aspects of this study were related first of all to the enrolment of the patients, taking into account that a baseline randomization was not well stratified.

The first method used to collect DNA samples using buccal swabs was much more appreciated by the patients, or by their parents because it was a painless procedure. This non-invasive technique is more time consuming in terms of training of physicians (dermatologist or pediatrician) to properly collect the swabs, without contaminating the sterile swab by hand or by other objects.

Moreover, another issue was related to the sample collection due to procedural defects during the sample collection (no sufficient DNA or contamination) or during the storage or transport of the samples. The DNA extraction and genotyping for FLG were elements of difficulty. The sample quality may vary due to procedural defects during the collection (insufficient DNA or contamination), during the storage or the transportation of samples.

Collection efficiency was assessed for the first few samples and required adjustments have been made, including the resampling of the patient. We have tried to reduce as much as possible the time between sample collection and DNA extraction.

On the contrary, the heterozygote for a loss-of-function mutation might carry an expanded exon 3 on the other allele, lessening the overall effect of the mutation (17).

Filaggrin skin expression could be modulated by the atopic inflammatory response mediated by the cytokines interleukin (IL)-4 and IL-13 $(22,23)$. The frequency of FLG mutations in healthy controls compared with atopic patients, can also be a limitation to current approaches. Another limitation is the presence of FLG mutations associated with higher disease severity $(24,25)$ inducing an overestimation of FLG mutations in AD which can be hard to identify.

A very recent Spanish study, performed on $111 \mathrm{AD}$ patients and 103 controls, searching for three FLG null mutations (R501X, 2282del4, and R2447X) found that combined mutation incidence was $1.9 \%$ in the healthy group and $12.6 \%$ in the AD group, and thus 9.9\% R501X, 2.7\% R2447X and $1.8 \%$ 2282del4, respectively (26).

A Croatian study published that combined mutation was found in $4 \%$ in the $\mathrm{AD}$ group, no mutations in the control-group and FLG mutations in AD patients being recorded: $3 \%$ -2282del4 and 1\% -R501X (27).

The possibility of a donor being apparently healthy while in fact affected by AD could not be excluded even though all patients with an uncertain diagnosis were replaced.

Filaggrin polymorphism is considered a major risk factor for AD. The variability of reported prevalence in different geographic areas is still controversial.

Studies on loss of function of the filaggrin gene are needed to elucidate the risks related to epidermal barrier defects involved in atopic diseases, to elucidate the pathogenic mechanisms and trigger factors, and to find new therapies for the disease.

The used methodology in this study may be adjusted according to future scientific advancements and to the evolution of new projects. More data and studies are needed to conclude the importance of FLG loss-of-function mutations in Romanian patients diagnosed with AD.

Genetic factors do not have a unique crucial role in $\mathrm{AD}$; therefore, environmental factors certainly play an important role in this disease, but the precise part that these components trigger an increasing risk of AD during childhood is still to be debated.

\section{Acknowledgements}

Not applicable.

\section{Funding}

No funding was received.

\section{Availability of data and materials}

The datasets used and/or analyzed during the current study are available from the corresponding author on reasonable request.

\section{Authors' contributions}

AEC and AM were involved in the acquisition of the data, analyzed and interpreted the patient data regarding the medical interview, and collected DNA samples from the patients. RP and LB performed the DNA extraction and FLG genotyping, and were major contributors in the writing of the manuscript. AEC, LF and DA conceived the study and designed the research, performed the analysis and interpretation of the data, were involved in the drafting of the manuscript, and revised the manuscript critically for important intellectual content. All authors contributed in the drafting and revising of the manuscript, gave final approval of the version to be published, and agree to be accountable for all aspects of the study.

\section{Ethics approval and consent to participate}

The reported study received ethics approval and consent by the institutional/national Ethics Committee of 'Grigore T. Popa' University of Medicine and Pharmacy, Iasi, Romania (Committee Reference no. 22168 of 10.10.2019). In accordance with the Declaration of Helsinki for all research involving human subjects, written informed consent for participation in the study and use of their tissue/blood/saliva sample (DNA sample) was obtained from all participants (or their parent or legal guardian in the case of children under 18 years of age).

\section{Patient consent for publication}

Not applicable.

\section{Competing interests}

The authors declare that they have no competing interests. 


\section{References}

1. Naldi L, Parazzini F and Gallus S; GISED Study Centers: Prevalence of atopic dermatitis in Italian school children: Factors affecting its variation. Acta Derm Venereol 89: 122-125, 2009.

2. Grize L, Gassner M, Wüthrich B, Bringolf-Isler B, Takken-Sahli K, Sennhauser FH, Stricker T, Eigenmann PA and Braun-Fahrländer C; Swiss Surveillance Programme on Childhood Allergy and Respiratory symptoms with respect to Air Pollution (SCARPOL) team: Trends in prevalence of asthma, allergic rhinitis and atopic dermatitis in 5-7-year old Swiss children from 1992 to 2001. Allergy 61: 556-562, 2006.

3. Solomon I, Ilie MA, Draghici C, Voiculescu VM, Caruntu C, Boda D and Zurac S: The impact of lifestyle factors on evolution of atopic dermatitis: An alternative approach. Exp Ther Med 17: 1078-1084, 2019.

4. Mischke D, Korge BP, Marenholz I, Volz A and Ziegler A: Genes encoding structural proteins of epidermal cornification and S100 calcium-binding proteins form a gene complex ('epidermal differentiation complex') on human chromosome 1q21. J Invest Dermatol 106: 989-992, 1996.

5. Mildner M, Jin J, Eckhart L, Kezic S, Gruber F, Barresi C, Stremnitzer C, Buchberger M, Mlitz V, Ballaun C, et al Knockdown of filaggrin impairs diffusion barrier function and increases UV sensitivity in a human skin model. J Invest Dermatol 130: 2286-2294, 2010.

6. Barresi C, Stremnitzer C, Mlitz V, Kezic S, Kammeyer A, Ghannadan M, Posa-Markaryan K, Selden C, Tschachler E and Eckhart L: Increased sensitivity of histidinemic mice to UVB radiation suggests a crucial role of endogenous urocanic acid in photoprotection. J Invest Dermatol 131: 188-194, 2011.

7. Compton JG, DiGiovanna JJ, Johnston KA, Fleckman P and Bale SJ: Mapping of the associated phenotype of an absent granular layer in ichthyosis vulgaris to the epidermal differentiation complex on chromosome 1. Exp Dermatol 11: 518-526, 2002.

8. Sandilands A, O'Regan GM, Liao H, Zhao Y, TerronKwiatkowski A, Watson RM, Cassidy AJ, Goudie DR, Smith FJD, McLean WH and Irvine AD: Prevalent and rare mutations in the gene encoding filaggrin cause ichthyosis vulgaris and predispose individuals to atopic dermatitis. J Invest Dermatol 126: 1770-1775, 2006.

9. Brown SJ, Relton CL, Liao H, Zhao Y, Sandilands A, Wilson IJ, Burn J, Reynolds NJ, McLean WH and Cordell HJ: Filaggrin null mutations and childhood atopic eczema: A population-based case-control study. J Allergy Clin Immunol 121: 940-946.e3, 2008.

10. Palmer CN, Irvine AD, Terron-Kwiatkowski A, Zhao Y, Liao H, Lee SP, Goudie DR, Sandilands A, Campbell LE, Smith FJD, et al: Common loss-of-function variants of the epidermal barrier protein filaggrin are a major predisposing factor for atopic dermatitis. Nat Genet 38: 441-446, 2006.

11. Smith FJ, Irvine AD, Terron-Kwiatkowski A, Sandilands A, Campbell LE, Liao H, Evans AT, Goudie DR, Lewis-Jones S, Arseculeratne G, et al: Loss-of-function mutations in the gene encoding filaggrin cause ichthyosis vulgaris. Nat Genet 38: 337-342, 2006.

12. Sandilands A, Terron-Kwiatkowski A, Hull PR, O'Regan GM, Clayton TH, Watson RM, Carrick T, Evans AT, Liao H, Zhao Y, et al: Comprehensive analysis of the gene encoding filaggrin uncovers prevalent and rare mutations in ichthyosis vulgaris and atopic eczema. Nat Genet 39: 650-654, 2007.

13. Fallon PG, Sasaki T, Sandilands A, Campbell LE, Saunders SP, Mangan NE, Callanan JJ, Kawasaki H, Shiohama A, Kubo A, et al: A homozygous frameshift mutation in the mouse Flg gene facilitates enhanced percutaneous allergen priming. Nat Genet 41: 602-608, 2009 .
14. McLean WH: Filaggrin failure - from ichthyosis vulgaris to atopic eczema and beyond. Br J Dermatol 175 (Suppl 2): S4-S7, 2016.

15. Weidinger S, O' Sullivan M, Illig T, Baurecht H, Depner M, RodriguezE, Ruether A, Klopp N, Vogelberg C, Weiland SK, et al: Filaggrin mutations, atopic eczema, hay fever, and asthma in children. J Allergy Clin Immunol 121: 1203-1209.e1, 2008.

16. Kezic S, Kemperman PM, Koster ES, de Jongh CM, Thio HB, Campbell LE, Irvine AD, McLean WH, Puppels GJ and Caspers PJ: Loss-of-function mutations in the filaggrin gene lead to reduced level of natural moisturizing factor in the stratum corneum. J Invest Dermatol 128: 2117-2119, 2008.

17. O'Regan GM, Kemperman PM, Sandilands A, Chen H, Campbell LE, Kroboth K, Watson R, Rowland M, Puppels GJ, McLean WH, et al: Raman profiles of the stratum corneum define 3 filaggrin genotype-determined atopic dermatitis endophenotypes. J Allergy Clin Immunol 126: 574-580, 2010.

18. Irvine AD, McLean WHI and Leung DY: Filaggrin mutations associated with skin and allergic diseases. N Engl J Med 365: 1315-1327, 2011

19. Chiriac A, Foia L, Gorduza VE, Chiriac AE, Uliliuc T, Kezic S and Solovan C: The puzzled low prevalence of atopic dermatitis in kindergarten children in Romania. Pediatr Allergy Immunol 25: 96-97, 2014.

20. Hannifin JM and Rajka G: Diagnostic features of atopic dermatitis. Acta Derm Venereol 92: 44-47, 1980.

21. Holm EA, Wulf HC, Thomassen L and Jemec JB: Instrumental assessment of atopic eczema: Validation of transepidermal water loss, stratum corneum hydration, erythema, scaling, and edema. J Am Acad Dermatol 55: 772-780, 2006.

22. Howell MD, Kim BE, Gao P, Grant AV, Boguniewicz M, Debenedetto A, Schneider L, Beck LA, Barnes KC and Leung DY: Cytokine modulation of atopic dermatitis filaggrin skin expression. J Allergy Clin Immunol 120: 150-155, 2007.

23. Spergel JM: From atopic dermatitis to asthma: The atopic march. Ann Allergy Asthma Immunol 105: 99-106; quiz 107-109, 117, 2010.

24. Gruber R, Janecke AR, Grabher D, Horak E, Schmuth M and Lercher P: Lower prevalence of common filaggrin mutations in a community sample of atopic eczema: Is disease severity important? Wien Klin Wochenschr 122: 551-557, 2010.

25. Wollenberg A, Oranje A, Deleuran M, Simon D, Szalai Z, Kunz B, Svensson A, Barbarot S, von Kobyletzki L, Taieb A, et al: ETFAD/EADV Eczema task force 2015 position paper on diagnosis and treatment of atopic dermatitis in adult and paediatric patients. J Eur Acad Dermatol Venereol 30: 729-747, 2016.

26. González-Tarancón R, Sanmartín R, Lorente F, SalvadorRupérez E, Hernández-Martín A, Rello L, Puzo J and Gilaberte Y: Prevalence of FLG loss-of-function mutations R501X, 2282del4, and R2447X in Spanish children with atopic dermatitis. Pediatr Dermatol 37: 98-102, 2020.

27. Jurakic Toncic R, Kezic S, Jakasa I, Ljubojevic Hadzavdic S, Balic A, Petkovic M, Pavicic B, Zuzul K and Marinovic B: Filaggrin loss-of-function mutations and levels of filaggrin degradation products in adult patients with atopic dermatitis in Croatia. J Eur Acad Dermatol Venereol 34: 1789-1794, 2020.

This work is licensed under a Creative Commons Attribution-NonCommercial-NoDerivatives 4.0 International (CC BY-NC-ND 4.0) License. 